Sharif University of Technology
Scientia Iranica
SCIENTIA
IRAN I CA
http://scientiairanica.sharif.edu

\title{
Wall and bed shear force in rectangular open channels
}

\author{
S. Tavakkol ${ }^{\mathrm{a}, \mathrm{b}, *}$ and A.R. Zarrati ${ }^{\mathrm{b}}$ \\ a. Department of Civil and Environmental Engineering, University of Southern California, 920 Downey Way, Los Angeles, CA \\ 90089, USA. \\ b. Department of Civil and Environmental Engineering, Amirkabir University of Technology, 424 Hafez St., Tehran, Iran.
}

Received 14 September 2016; received in revised form 12 January 2017; accepted 18 April 2017

\section{KEYWORDS}

Open channel flow;

Velocity distribution;

Shear force;

Secondary flow;

Dip phenomenon.

\begin{abstract}
This paper investigates the effects of velocity gradients and secondary currents on distribution of the shear force between the walls and bed of rectangular open channels. This paper shows that neglecting the effect of secondary currents and assuming zeroshear division lines do not yield acceptable results. Then, accordingly, a method was introduced to determine the percentage of the total shear force acting on the walls and bed of rectangular open channels, taking both velocity gradients and secondary currents into account. Using the channel bisectors, along which there is no secondary flows effect, and orthogonal trajectories to isovels, along which there is no shear stress, the channel's crosssection was divided into three major subsections: bed, wall, and shared areas. The geometry of each subsection was derived with respect to the location of the maximum velocity. The share of the bed and wall shear forces from the shared area were calculated afterwards. The results for bed and walls shear forces are in agreement with the experimental data containing an average relative error of less than $5 \%$ for regular flows and the flows carrying suspended sediment. This method also provides a physics-driven range for the wall and bed shear forces, which nicely covers the experimental data.

(C) 2018 Sharif University of Technology. All rights reserved.
\end{abstract}

\section{Introduction}

In a uniform flow, total shear force acting on the wetted perimeter can be calculated in terms of bed slope, hydraulic radius, and fluid density. While measuring the total shear force is useful in many engineering problems such as loss estimations in hydraulic structures [1], there is a need for the distribution of the shear force on the wetted perimeter in many more problems. Despite recent advances and applications of modern techniques such as soft computing [2-4] and entropy concept [5], calculating the portion of the shear force acting on side walls and the channel bed is a subject of research even after decades [6-8]. Calculating the shear force

*. Corresponding author. Tel.: +1213 3090723 E-mailaddress: tavakkol@usc.edu (S. Tavakkol) acting on the bed and channel walls is important in hydraulic engineering studies. For example, the bed mean shear stress is required to calculate the bed load [9], and the wall mean shear stress is required to study bank erosion, channel migration, and river morphology. Moreover, the knowledge of shear force ratio is required in laboratory flume studies. Given that empirical results of flume studies are often subject to sidewall friction effects, a procedure based on the shear stress ratio can be used to remove these effects, referred to as the sidewall correction procedure $[10,11]$.

The present paper introduces a method to determine the percentage of the total shear force acting on the walls $\left(\% S F_{w}\right)$ or bed $\left(\% S F_{b}\right)$ based on the location of the maximum velocity on the centerline of a rectangular channel cross-section. Incorporating the location of the maximum velocity gives an advantage to the proposed method compared to the previous ones, making it more accurate for the cases in which 
this location can be obtained. For channels not open to measurements, the location of the maximum velocity can still be found through the instrumentality of empirical equations.

Among different methods for local shear stress calculation, a series of simple, yet popular, methods exist that rely on splitting the channel cross-section into several subsections. In these methods, it is assumed that the shear force along its corresponding wetted perimeter balances the weight component of the fluid inside each subsection. Leighly [12] employed this idea for the first time to estimate the shear stress distribution. He mapped the channel cross-section by lines of constant velocity (isovels) and their orthogonal curves. He then defined the subsections as the area between two consecutive orthogonal curves. Since the velocity gradient across orthogonal curves is zero, these curves are assumed to be "surfaces of zero shear"; however, the presence of secondary flows causes momentum exchange and, consequently, resistance force along these surfaces. The interaction among main flow, secondary flows, and shear stress distribution is well investigated by Chiu and Chiou [13] and Yang [14].

Following Leighly [12], several researchers [15-17] developed methods for predicting shear stress distribution considering zero-shear division lines on the channel cross-section. According to this assumption, the crosssection of a channel can be separated by two zero-shear division lines into three parts: bed area, left wall area, and right wall area. In this assumption, the component of the fluid weight in each area is assumed to be balanced by the resistance of the channel boundaries in contact with that area.

Yang and Lim [8] proposed that the surplus energy of a unit volume of fluid in a three-dimensional channel is dissipated at the "closest" wall boundary. Based on this assumption, they developed a linear implicit equation for the zero-shear division lines. Later, Gou and Julien [6,11] employed the differential form of momentum and mass conservation equations to formulate shear force distribution between walls and bed of a rectangular channel. Deriving this formulation is straightforward using the integral form of these conservation equations. Employing the Schwarz-Christoffel transformation, they formulated the orthogonal curve to isovels drawn from the channel bottom corner and assumed it as a zero-shear division line. Their proposed equation, as stated by the authors, did not capture the dip phenomenon, and implied that the maximum velocity occurred on the water surface. Their assumed curve as an orthogonal trajectory did not conform with the experimental data of Nezu and Nakagawa [18]. Therefore, Gou and Julien [6] introduced two lumped empirical correction factors to correct the error caused by neglecting the secondary currents and deviation of division curves from the experiments.
In the present paper, firstly, the importance of different terms of momentum equation on a control volume surrounded by an isovel and a control volume between two curves perpendicular to the isovels is explained. The possibility of neglecting the secondary currents is examined next, showing that assuming zeroshear division lines is not acceptable. Finally, a new method is introduced to calculate the percentage of shear force acting on the channel bed and sidewalls. The proposed subdividing method is applied to uniform rectangular open channels with fully developed turbulent flows. However, the general concept can be extended to other cross-sectional shapes.

\section{Theoretical consideration}

For an arbitrary control volume on the cross-section of a rectangular channel (Figure 1), assuming a steady uniform flow, the continuity and momentum equations can be written as follows:

$$
\begin{aligned}
& \oint \rho(V . n) d l=0, \\
& \rho g A S+\oint \tau_{n x} d l-\oint \rho u(V . n) d l=0,
\end{aligned}
$$

where $\rho$ is density and $A$ is area of the control surface on $y-z$ plane, $g$ is gravitational acceleration, $S$ is channel slope, $V$ is velocity component of flow on $y-z$ plane, $\mathbf{n}$ is unit normal vector pointing outside of the control volume, $l$ is length along the boundary of surface, and $\tau_{n x}$ is shear stress in the flow direction $x$ applying on the plane perpendicular to $\mathbf{n}$. Flow velocity components $u, \nu$, and $w$ are in longitudinal, lateral, and vertical (i.e., $x, y$, and $z$ ) directions, respectively.

For a control volume surrounded by an isovel (i.e., constant $u$ ), the momentum equation (Eq. (2)) can be rewritten as follows:

$$
\rho g A S+\oint \tau_{n x} d l-u \oint \rho(V . n) d l=0 .
$$

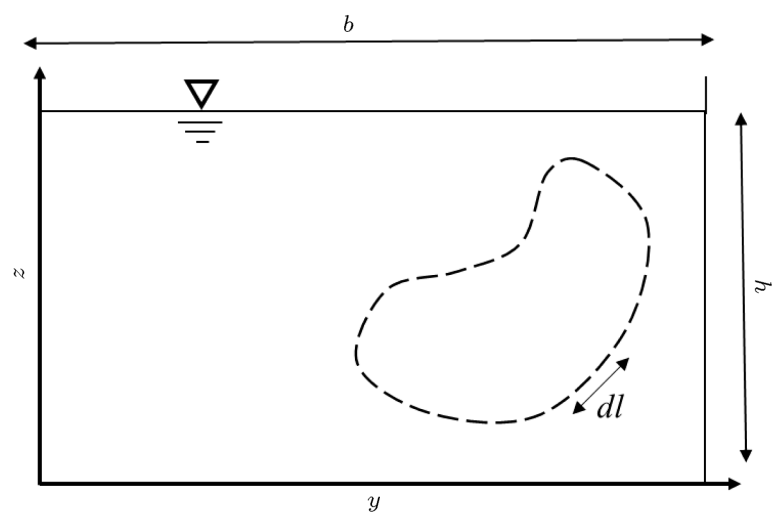

Figure 1. An arbitrary control volume in a channel cross-section. 


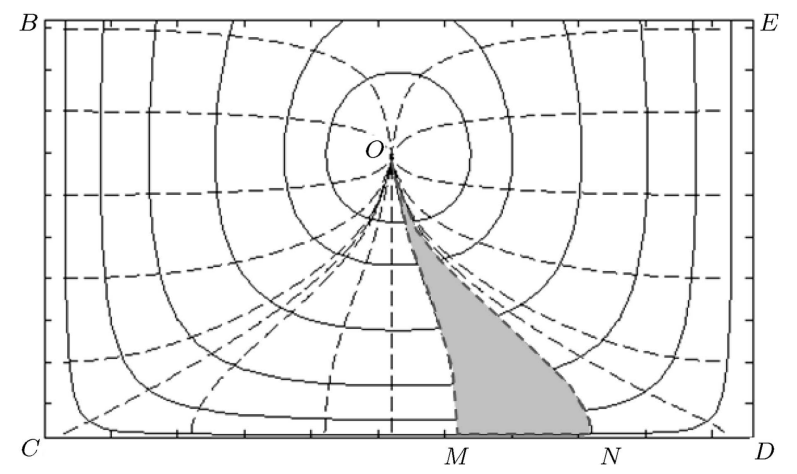

Figure 2. Isovels and orthogonal curves for $b / h=2$ (isovels are calculated following Chiu and Hsu, 2006 [26]).

Considering Eq. (1), the third term of Eq. (3), which represents the effect of secondary flows, vanishes and Eq. (3) reduces to:

$$
\rho g A S+\oint \tau_{n x} d l=0
$$

Eq. (4) shows that the gravity component of a control volume limited to an isovel is balanced only by the shear stress acting on its surface due to velocity gradients. On the other hand, if the momentum equation is applied to a control volume surrounded by two curves (polygon MNOM in Figure 2), which are orthogonal to isovels, one can write:

$$
\rho g A_{M N O M} S+\oint_{M N O M} \tau_{n x} d l-\oint_{M N O M} \rho u(V . n) d l=0
$$

where $A_{M N O M}$ is the area of the control volume surrounded by the polygon $M N O M$, and both integrals are calculated along the same polygon. The first integral on the left-hand side of Eq. (5) can be calculated on subsections of the polygon MNOM as follows:

$$
\int_{M N O M} \tau_{n x} d l=\int_{M N} \tau_{n x} d l+\int_{N O} \tau_{n x} d l+\int_{O M} \tau_{n x} d l .
$$

$\tau_{n x}$ can be assumed as follows:

$$
\tau_{n x}=\rho\left(\nu_{t}+\nu\right) \frac{\partial u}{\partial n},
$$

where $\nu$ and $\nu_{t}$ are kinematic and turbulent viscosity of water, respectively. Since NO and OM are orthogonal to isovels, $\partial u / \partial n$ is zero along them; therefore, integrals $\int_{N O}$ and $\int_{O M}$ are zero, too. Considering the average shear stress on MN as $\tau_{M N}$, its length as $\delta P_{M N}$, and Eq. (5), we can derive:

$$
\left|\tau_{M N}\right|=\frac{1}{\delta P_{M N}}\left(\rho g A_{M N O M} S-\oint_{M N O M} \rho u(V . n)\right) d l .
$$

For a known 3D velocity field, one can calculate the shear stress distribution on the channel boundary employing Eq. (8); however, usually, all 3 velocity components are not known. Note that viscosity does not show up in Eq. (8); however, its effects are considered through the velocity field.

Based on the above discussion, shear resistance cannot be neglected neither on a control volume surrounded by isovels nor surrounded by orthogonal curves to isovels. Thus, it seems unlikely to find a series of zero shear curves in the cross-section of an open channel. The channel centerline, however, is an exception at which both the shear resistance caused by secondary currents and velocity gradient are zero due to symmetry. If secondary currents are neglected, despite their indisputable effect, Eq. (8) becomes:

$$
\left|\tau_{M N}\right|=\frac{\rho g A_{M N O M} S}{\delta P_{M N}}
$$

Eq. (9) describes Leighly's assumption, which is proved not be accurate. Guo and Julien [6] employed the same assumption to propose a first approximation for mean shear stress on the channel bed and walls.

Regardless of the secondary currents, the distribution of the main velocity, $u$, should be known to calculate the orthogonal curves to isovels and, consequently, the distribution of shear stress on the channel boundary. It is well known that, in an open channel, the maximum velocity may occur at a point below the water surface [19]. The location of the maximum velocity is important, because it is the point of concurrency of the isovel orthogonal trajectories.

Several researchers have proposed methods to calculate the velocity distribution in an open channel. The method of Maghrebi and Rahimpour [20], similar to that of Houjou and Ishii [21], predicts the location of the maximum velocity to be below the water surface for $b / h<2$, where $b$ is the channel width and $h$ is the flow depth. This result is due to the fact that an open channel with $b / h=2$ is half of a square conduit, in which the velocity profile is symmetrical in $y$ and $z$ directions [21]. De Cacqueray et al. [22], who examined the formulation of Guo and Julien [6] using a detailed computational fluid dynamic simulation, reported that the maximum velocity occurs on the flow surface for a channel with $b / h=2$ for their numerical experiments. However, it is widely reported that the dip phenomenon is observed for channels with $b / h<5$ [23-25].

The velocity distribution proposed by Chiu and $\mathrm{Hsu}$ [26] is employed in the present paper to calculate the shear force distribution. Chiu and Hsu [26] presented an entropy-based approach to calculate the velocity distribution. This method relies on a parameter which could be found according to various hydraulic parameters of the channel, such as the ratio of the average velocity to the maximum velocity and 
the location of the maximum velocity. The location of the maximum velocity can be measured or calculated through the method from Yang et al. [27] as follows:

$$
\frac{\varepsilon}{h}=1-\frac{1}{1+1.3 e^{-b / 2 h}}
$$

in which $\varepsilon$ is the depth of the maximum velocity.

The isovels and their orthogonal curves for a channel with $b / h=2$ calculated by Chiu and Hsu [26] method based on Eq. (10) are shown in Figure 2. The slope of the isovel orthogonal trajectories at any point can be calculated from $\tan (\theta)=(\partial u / \partial z) /(\partial u / \partial y)$. Starting from a certain point on the boundary and following the slope, the orthogonal trajectories can be drawn. If the momentum exchange generated by the secondary currents on the isovel orthogonal curves is neglected, the gravity component of the mass surrounded by the closed polygon BOEB (Figure 2) remains unbalanced, which leads to the underestimation of the total shear force on the channel boundary.

Figure 3 compares the estimation of Eq. (9) for $\% S F_{b}$ with the empirical equation presented by Knight et al. [28]. As is illustrated in Figure 3, neglecting the secondary currents leads to underestimating the bed shear force and is not acceptable. The location of the maximum velocity moves toward the flow surface in wider channels; therefore, predictions of Eq. (9) become more accurate for larger $b / h$.

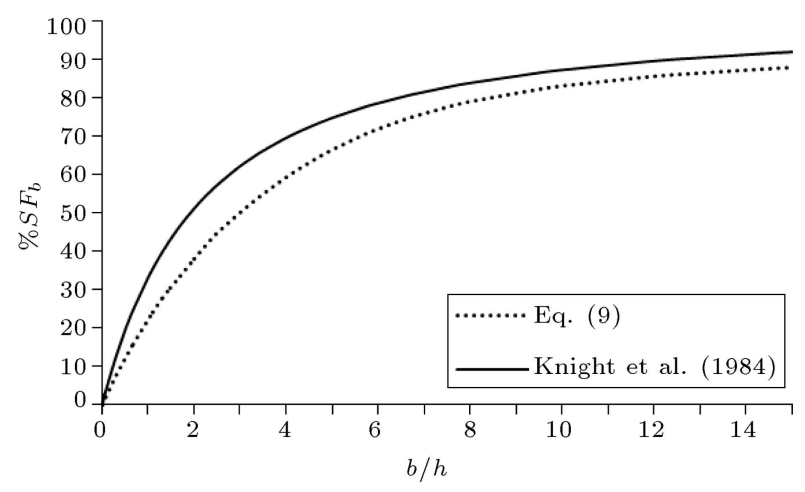

Figure 3. Comparison of $\% S F_{b}$ predicted by neglecting secondary currents with the empirical equation presented by Knight et al. (1984) [28].

\section{The present methodology}

Eq. (8) can be employed to calculate the mean shear on the bed and sidewalls of an open channel if the effect of the secondary currents can be calculated. If the orthogonal trajectories to the isovels are drawn from the channel corners, they will intersect in the maximum velocity location $\left(P_{2}\right.$ curves in Figure 4$)$. The weight component of water in the area limited to $P_{2}$ curves and channel bed, $A_{b \text { min }}$, must be less than the shear force acting on the bed, since $P_{2}$ curves are orthogonal to isovels, with no velocity gradients and, consequently, no shear stress acting on them. However, there are secondary flows passing across these curves that cause momentum exchange. Secondary currents enter this area close to the channel centreline [19], where the main flow velocity is maximum. Due to the continuity, the flow entering this area close to the channel centerline leaves this area to locations where flow velocity is smaller. Therefore, the acting force due to the momentum transfer is in the flow direction. It will be shown later that the measured bed shear force is in excess of the weight component of the water in this area $\left(A_{b \min }\right)$.

Gessner and Jones [29] suggested that the secondary flow cells in the corners of rectangular ducts are divided by the corner bisector. Experimental data presented by Tominaga et al. [30] show that secondary flows are strong in a region within a distance equal to $0.65 \mathrm{~h}$ from the channel bottom corners. Zheng and Jin [31] and Jin et al. [32] assumed the same structure for corner secondary currents as Gessner [29] did in rectangular open channels, with the boundary of $y / h=0.65$ and $z / h=0.65$. It was assumed that the secondary flow cells have negligible effect on the flow mechanism out of this boundary [32]. The effective area of the secondary currents on the wall side can be, therefore, limited to the sidewall, bisector and a vertical line at $y / h=0.65$ (region CJKBC, Figure 4). The weight component of water in this region must be less than the wall shear force, because it is assumed that secondary flows do not pass through the boundaries of this area; therefore, there is no momentum exchange due to secondary currents across these boundaries. However, there could be momentum exchange due to

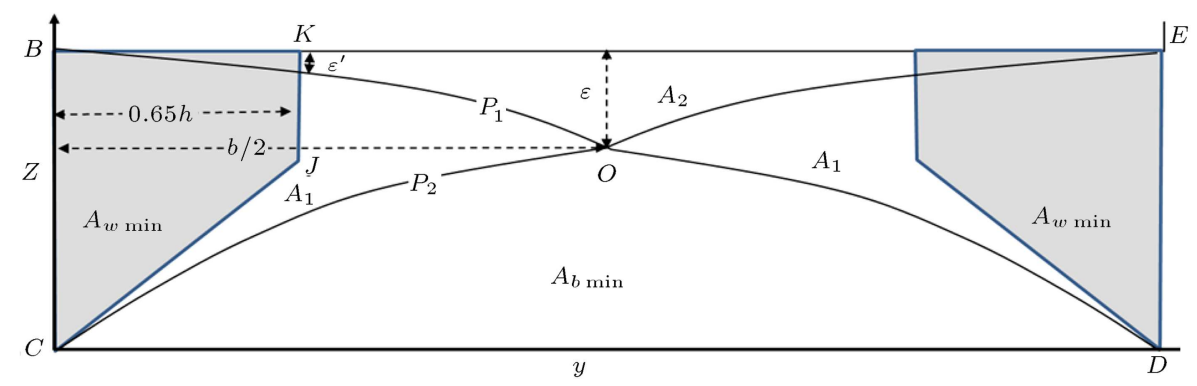

Figure 4. Dividing the channel cross-section by bisectors $(C J)$ and isovels orthogonal curves $\left(P_{1}\right.$ and $\left.P_{2}\right)$. 
the shear force in the flow. Since, in general, flow velocity decreases toward the sidewall, the shear force acting on the CJK is in the flow direction and its value is added to the weight component of CJKBC and does help balance it. This area, called $A_{w \text { min }}$, can be easily calculated as follows:

$$
\begin{aligned}
A_{w \min } & =2 \times\left(\frac{h+0.35 h}{2} \times 0.65 h\right) \\
& =0.65\left(1.35 h^{2}\right) \approx 0.8 h^{2} \quad \text { for } \quad b / h>1.3 .
\end{aligned}
$$

In channels with $b / h<1.3$, the corner bisectors intersect below $z=0.65 h$. This case will be discussed later.

According to these assumptions, CJK and $P_{2}$ in Figure 4 split the minimum share of sidewalls and bed from total shear force respectively. However, the weight component of the area between these two curves is assumed to be balanced partially by shear force acting on the sidewalls and partially with the shear force acting on the bed. The shared areas are divided into $A_{1}$ and $A_{2}$ by $P_{1}$ curve (Figure 4 ). $P_{1}$ is the orthogonal curve to isovels drawn from upper corners of the channel cross-section. Let the share of the sidewalls from weight components of $A_{1}$ and $A_{2}$ be denoted by $\eta_{1}$ and $\eta_{2}$, respectively. One can, therefore, calculate $\% S F_{w}$ and $\% S F_{b}$ as follows:

$$
\begin{aligned}
\% S F_{W} & =\frac{2 \tau_{w} h}{\rho g A S} 100 \% \\
& =\frac{100 \%}{A_{t}}\left(A_{w \min }+\eta_{1} A_{1}+\eta_{2} A_{2}\right) \\
\% S F_{b} & =\frac{\tau_{b} b}{\rho g A S} 100 \% \\
& =\frac{100 \%}{A_{t}}\left(A_{b \min }+\left(1-\eta_{1}\right) A_{1}+\left(1-\eta_{2}\right) A_{2}\right)
\end{aligned}
$$

where $\tau_{b}$ and $\tau_{w}$ are mean bed and wall shear stresses, respectively, and $A_{t}$ is the channel cross-sectional area. To determine $\eta_{1}$ and $\eta_{2}, A_{1}$ and $A_{2}$ must be calculated first. To calculate these areas, equations for $P_{1}$ and $P_{2}$ must be known. $P_{1}$ can be approximated by a parabola. $P_{1}$ passes through $(0, h)$ and $(b / 2, h-\varepsilon)$. It is also perpendicular to the channel sidewall as an isovel; therefore, its derivative is zero in $(0, h)$. The equation of $P_{1}$ can therefore be written as follows:

$$
z=-\frac{\varepsilon}{(b / 2)^{2}} y^{2}+h .
$$

Similarly, $P_{2}$ passes through $(0,0)$ and $(b / 2, h-\varepsilon)$. At the corner, $P_{2}$ must be perpendicular to both the channel bed and wall, which is not possible. To remove this conflict, following Chiu and Chiou [13], the channel corner is assumed as a quarter circle; therefore, the slope of $P_{2}$ in the corner is assumed equal to 1 .

Contrary to $P_{1}, P_{2}$ cannot be approximated by a parabola. A parabolic approximation significantly deviates from $P_{2}$ for larger $b / h$ and may even exit the water surface. To avoid this problem, $P_{2}$ is approximated by a parabola starting on the channel corner and a straight line following the parabola at a point to be determined, say $\left(y_{i}, z_{i}\right)$. Considering these boundary conditions, $P_{2}$ can be expressed as follows:

$$
\begin{array}{ll}
z=a^{\prime} y^{2}+b^{\prime} y+c^{\prime} & y \leq y_{i}, \\
z=a^{\prime \prime} y+b^{\prime \prime} & y>y_{i},
\end{array}
$$

where:

$$
\begin{aligned}
& a^{\prime}=\frac{z_{i}-y_{i}}{y_{i}^{2}} ; \quad b^{\prime}=1 ; \quad c^{\prime}=0 ; \\
& a^{\prime \prime}=\frac{h-\varepsilon-z_{i}}{b / 2-y_{i}} ; \quad b^{\prime \prime}=h-\varepsilon-a^{\prime \prime} \frac{b}{2} .
\end{aligned}
$$

The slopes of the parabola and the line are equal at their intersection point. This condition results in the following equation between $y_{i}$ and $z_{i}$ :

$$
y_{i}^{2}-\left(z_{i}+\frac{b}{2}+(h-\varepsilon)\right) y_{i}+z_{i} b=0 .
$$

For a given $z_{i}$, one can calculate $y_{i}$ from Eq. (16) and, consequently, the unknown coefficients $a^{\prime}, a^{\prime \prime}$, and $b^{\prime \prime}$. $z_{i}$ is found by employing the least squares regression between Eq. (15) and isovel orthogonal curves obtained based on Eq. (10). Plotting $z_{i} /(h-\varepsilon)$ against $b / h$ indicates that $z_{i} /(h-\varepsilon)$ is almost constant and can be assumed equal to 0.93 for different values of $b / h$ with about $2 \%$ averaged relative error. Figure 5 compares Eq. (15) with the Chiu and Hsu's [26] equations while assuming that $z_{i} /(h-\varepsilon)=0.93$.

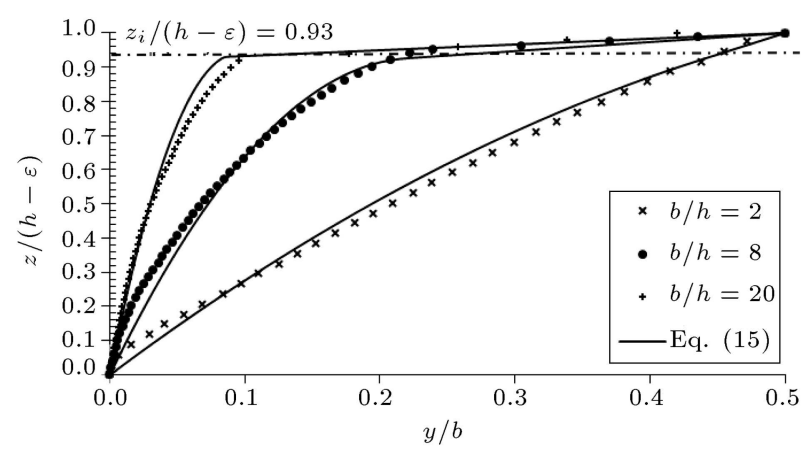

Figure 5. Comparison of Eq. (15) for $z_{i} /(h-\varepsilon)=0.93$ with numerical data calculated through Chiu and Hsu (2006) method [26]. 
According to the equations found for $P_{1}$ and $P_{2}$, $A_{1}$ and $A_{2}$ can be presented as follows:

$$
\begin{aligned}
& A_{1}=b h-A_{w \min }-A_{b \min }-A_{2}, \\
& A_{b \min }=2\left(\frac{a^{\prime} y_{i}^{3}}{3}+\frac{y_{i}^{2}}{2}+\frac{z_{i}+h-\varepsilon}{2}\left(\frac{b}{2}-y_{i}\right)\right), \\
& A_{2}=2\left(\frac{b}{6}\left(\varepsilon-\varepsilon^{\prime}\right)\right)=\frac{b}{3}\left(\varepsilon-\varepsilon^{\prime}\right),
\end{aligned}
$$

where $\varepsilon^{\prime}$ is the vertical distance between the intersection points of $P_{1}$ and CJK from the water surface (Figure 4). $\varepsilon^{\prime}$, shown in Figure 4, can be calculated from Eq. (16) as follows:

$$
\varepsilon^{\prime}=\frac{\varepsilon}{(b / 2)^{2}}(0.65 h)^{2}
$$

The theory can be extended to $b / h<1.3$ while considering necessary changes in the equations. For a channel with $b / h<1.3$, the bottom corner bisectors intersect at the channel axis in a point like $\mathrm{N}$, and two different patterns may occur (Figure 6). The maximum velocity may be below point N (Figure 6(a)), meaning that $h-\varepsilon<b / 2<0.65 h$. This pattern is similar to that of the channel cross-section with $b / h>1.3$; however, Eqs. (11) and (19) must be substituted by $A_{w \min }=b(h-b / 4)$ and $A_{2} \approx 0$, respectively. The maximum velocity may occur above point $\mathrm{N}$, which means that $b / 2<h-\varepsilon<0.65 h$ (Figure 6(b)). In this case, $A_{w \text { min }}$ is limited to $P_{2}$ and sidewall, and $A_{b \text { min }}$ is limited to the bisectors and channel bed and can, therefore, be easily calculated.

Employing Eq. (10) to calculate $\varepsilon$ shows that, in $b / h=1.16$, point $\mathrm{N}$ conforms to point $\mathrm{O}$. In this particular and interesting case, the bottom corner bisectors and $P_{2}$ conform and, therefore, can be assumed as actual division lines across which there is no shear stress due to velocity gradient, or no momentum exchange due to secondary currents. In this case, $\eta_{1}$ and $\eta_{2}$ are eliminated from Eq. (12), and $\% S F_{w}$ is equal to $\% 71$. Figure 7 compares this

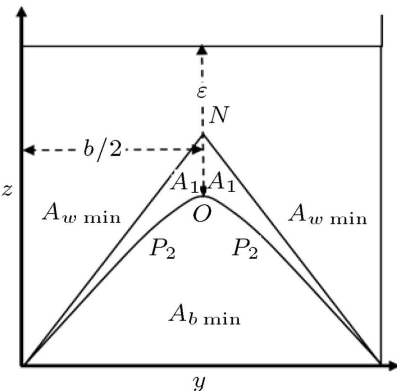

(a)

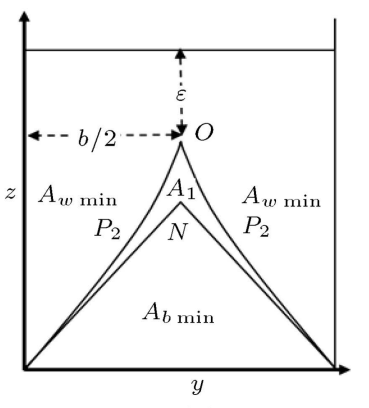

(b)
Figure 6. Possible cross-sectional division lines for $b / h<1.3$.

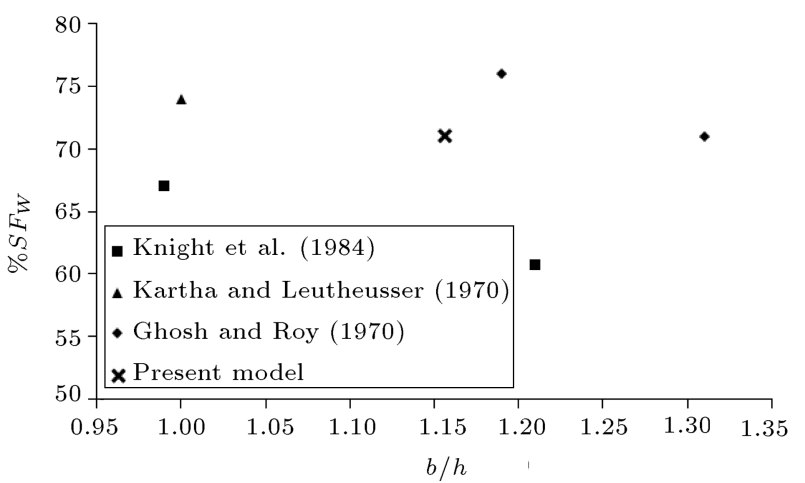

Figure 7. Comparison of $\% S F_{w}$ for $b / h=1.16$ with experimental results.

value with experimental results derived from different researches for $b / h$ values close to 1.16. As seen from this figure, $\% S F_{w}$ calculated from the present theory is surrounded by the experimental results. A Gaussian weighted average from the shown experimental results in Figure 7 gives $\% S F_{w}=69 \%$ for $b / h=1.16$ which is very close to the predicted value of the present model. However, estimation of Knight et al.'s [25] empirical equation and Guo and Julien [15] is $63.2 \%$ and $62.9 \%$, respectively.

\section{Calibration}

Figure 8 compares the result of Eq. (12) with experimental data presented by Knight et al. [28] from various researchers [33-39]. For $\eta_{1}=\eta_{2}=0$, Eq. (12) gives the minimum possible value of $\% S F_{w}$; for $\eta_{1}=\eta_{2}=1$, it gives the maximum possible value of $\% S F_{w}$. It can be seen in Figure 8 that the experimental data lie very well between the predicted minimum and maximum values for $\% S F_{w}$, which demonstrates the validity of dividing the channel sections into $A_{b \text { min }}$ and $A_{w \text { min }}$. Contrary to the coefficients used in previous related works, coefficients $\eta_{1}$ and $\eta_{2}$ are physically meaningful and limited to a range between 0 and 1 . In addition, considering the maximum and minimum values of

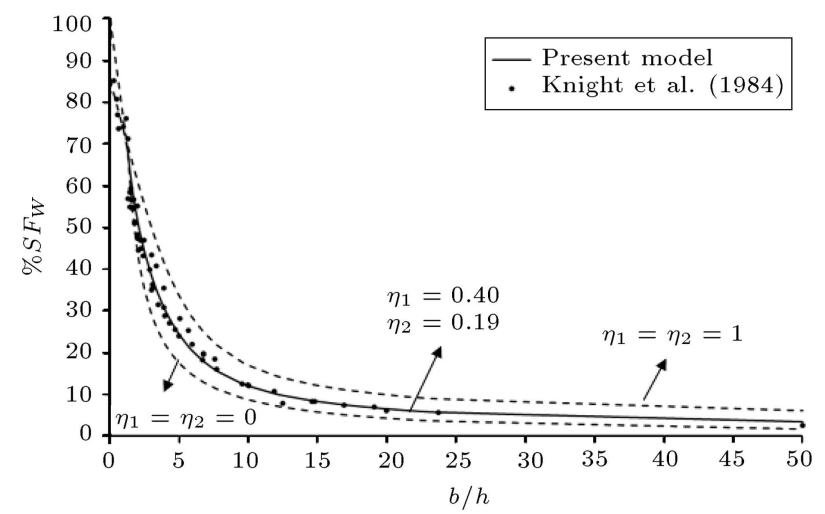

Figure 8. Comparison of the present model for $\% S F_{w}$ with experimental data by Knight et al. [28]. 
$\% S F_{w}$ illustrated in Figure 8, it can be seen that the present model is not significantly sensitive to the values of $\eta_{1}$ and $\eta_{2}$.

Employing the least squares method to estimate the share coefficients results in $\eta_{1}=0.40$ and $\eta_{2}=$ 0.19 with the coefficient of determination of $R^{2}=0.98$. According to these obtained values for $\eta_{1}$ and $\eta_{2}, \% S F_{w}$ can be calculated from:

$$
\% S F_{w}=\frac{100 \%}{b h}\left(A_{w \min }+0.40 A_{1}+0.19 A_{2}\right)
$$

where $A_{w \min }, A_{1}$, and $A_{2}$ are calculated from Eqs. (11), (17), and (19), respectively. In addition, $\% S F_{w}$ values can be read from Figure 9 given the values of $b / h$ and $\varepsilon / h$.

\section{Validation}

The presented model is validated with several sets of unseen data, and the results are compared with predictions of Guo and Julien [6]. In order to measure how well each equation functions, Mean Absolute Percentage Error (MAPE), Root-Mean-Square Error (RMSE), and Mean Signed Deviation (MSD) are utilized, which are defined as below:

$$
\begin{aligned}
& M A P E=\frac{100}{n} \sum_{i=1}^{n}\left|\frac{\hat{y}_{i}-y_{i}}{y_{i}}\right|, \\
& R M S E=\sqrt{\frac{1}{n} \sum_{i=1}^{n}\left(\hat{y}_{i}-y_{i}\right)^{2}},
\end{aligned}
$$

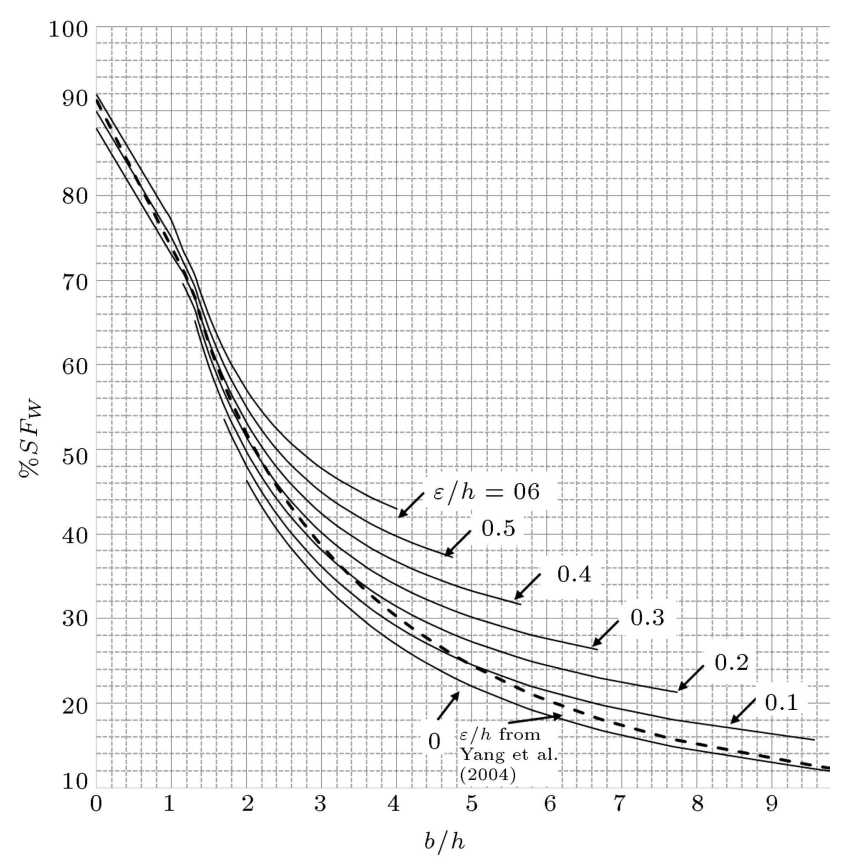

Figure 9. Values of $\% S F_{w}$ for different values of $b / h$ and $\varepsilon / h$.

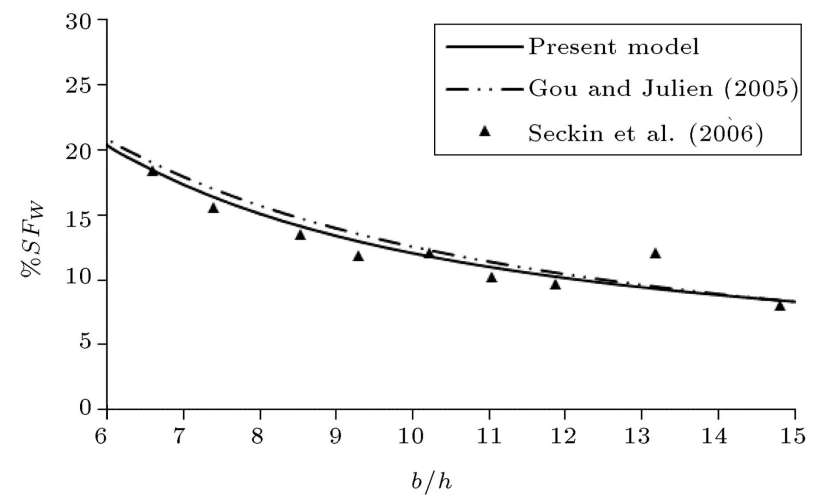

Figure 10. Comparison of the present model for $\% S F_{w}$ with unseen experimental data.

$$
M S D=\frac{1}{n} \sum_{i=1}^{n} \hat{y}_{i}-y_{i},
$$

where $\hat{y}$ is the predicted value of $y$, and $n$ is the number of data points. MAPE and RMSE are measures of prediction accuracy, while MSD determines if a prediction method tends to underestimate or overestimate the values.

Figure 10 compares the present model (Eq. (21)) with experimental data of wall shear force from Seckin et al. [40] which are neither included in the calibration of the present model, nor in calibration of Guo and Julien [6] model. By excluding the experiment with $b / h=13.18$ as an outlier, the MAPE for the present model is $4.7 \%$, while it is $7.7 \%$ for Gou and Julien [6]. The RMSE of the present model is 0.63 and its value for Guo and Julien's model is 1.05. The MSD for both models is positive, which means that our model and Gou and Julien's overestimate $\% S F_{w}$ with mean values of 0.46 and 0.93 , respectively. Eq. (21) is, therefore, in very good agreement with the experimental data of Seckin et al. [40].

Results of comparison of the present model (Eq. (21)) and Gou and Julien (2005) with experimental measurements of Xie [41] and Chanson [42] for $\% S F_{w}$ are shown in Table 1 . The MAPE for the present model is $8.6 \%$, while it is $9.3 \%$ for model of Gou and Julien [6].

Table 2 compares the result of Eq. (21) with experimental data of average bed shear velocity from experimental data of Coleman [43], Lyn [44,45], Muste and Patel [46], and predicted values by Gou and Julien [6]. The average bed shear velocity is calculated from $u_{* b}=\sqrt{\tau_{b} / \rho}$. In this table, $u_{* b, m}$ represents the measured values, $u_{* b, 21}$ is the results of Eq. (21), $\varepsilon$ is calculated from Eq. (10), and $u_{* b, G \& J}$ is the prediction of Gou and Julien [6]. It can be seen that the predicted values by the analytical models are slightly smaller than the measured values. This is due to the fact that the measured values are at a channel centreline; however, the predicated values are mean bed shear 
Table 1. Comparison of the present model and Gou and Julien (G\&J) [6] for $\% S F_{w}$ with experimental data of Xie [41] and Chanson [42].

\begin{tabular}{ccccc}
\hline & $\boldsymbol{b} / \boldsymbol{h}$ & Measured & G\&J & Eq. (21) \\
\hline Xie [41] & 3.2 & 36 & 36.40 & 36.62 \\
& & & & \\
Chanson & 3.24 & 37 & 36.05 & 36.25 \\
[42] & 5.32 & 19 & 23.35 & 22.98 \\
& 5.95 & 18 & 20.99 & 20.50 \\
MAPE & & & & \\
RMSE & & & 2.3 & 8.6 \\
MSD & & & 1.61 & 2.26 \\
\hline
\end{tabular}

velocities. The MAPE between results of Eq. (21) and experimental data is $3.23 \%$, while it is $3.48 \%$ for model of Gou and Julien [6].

Coleman $[43,47]$ reported the experiments conducted in a channel with $S=2 \times 10^{3}, b=35.6 \mathrm{~mm}$, and $Q=0.64 \mathrm{~m}^{3} / \mathrm{s}$, where $S$ is the bottom slope and $Q$ is the channel discharge. The experiments are carried out to investigate the effect of sediment suspension on a velocity profile. Coleman reported the location of maximum velocity in all experiments which can, therefore, be used directly in Eq. (21).

Table 3 compares the results calculated from the present method and model of Guo and Julien [6] with experimental data of Coleman [43]. In this table, $u_{* b, 21}$ is the result of $\mathrm{Eq}$. (21) by directly substituting $\varepsilon$ into the experiment. The total amount of sand suspended in the flow is given in this table in $\mathrm{Kg}$ as $M_{s}$. Moreover, the average volumetric suspended sediment concentration $(\varphi)$ of flow is calculated from available data in Coleman [43] and is provided in the table. It can be seen that the present model often predicts the average shear velocities to be equal to or slightly smaller than $u_{* b, m}$; however, $u_{* b, G \& J}$ is always larger than $u_{* b, m}$. The predicted mean values, as mentioned before, should be slightly smaller than the measured local values since the measurements are made at the channel centreline. The measured shear velocities are almost constant and equal to $4.1 \mathrm{~cm} / \mathrm{s}$, though the aspect ratio of the channel is not constant. This is because velocity profile in each experiment also changes due to different sediments in suspension. The results, therefore, show that the bed shear velocity of an open channel is not only a function of the channel aspect ratio, but also a function of the location of the maximum velocity. The present model successfully captures this fact. It is, therefore, concluded that the present model can be extended to other flow conditions if the location of maximum velocity is known. The model can be effectively used for the sidewall correction procedure in flume studies, because, normally, in these studies, some experimental information on flow is available which can help estimate $\varepsilon$. In cases where the pattern of the velocity distribution is different, e.g., in trapezoidal or vegetated channels, the idea of the current research is still applicable by modifying the boundaries of $A_{w \text { min }}$ and $A_{b \text { min. }}$. The equations for $P_{1}$ and $P_{2}$ might also change based on the velocity distribution.

It should be noted that the present method could be used as far as the location of maximum velocity, $\varepsilon$, is known. $\varepsilon$ may be available in experiments or can be calculated based on other hydraulic parameters of an open channel flow using a graph presented by Chiu and Hsu [26]. While the equations proposed in the present research are derived for rectangular open channels, the proposed method of dividing the channel cross-section into subsections can be developed for channels with other cross-sectional shapes. Knight et

Table 2. Comparison of the present model for bed shear velocities $(\mathrm{cm} / \mathrm{s})$ with experimental data.

\begin{tabular}{|c|c|c|c|c|c|c|}
\hline & & $S \times 10^{3}$ & $b / h$ & $\boldsymbol{u}_{* b, m}$ & $\boldsymbol{u}_{* b, 21}$ & $\boldsymbol{u}_{* b, G \& J}$ \\
\hline Coleman (1986) [43] & Run 1 & 2 & 2.07 & 4.1 & 4.08 & 4.14 \\
\hline \multirow{4}{*}{$\begin{array}{c}\text { Lyn } \\
(1986,2000) \\
{[44,45]}\end{array}$} & $\mathrm{C} 1$ & 2.06 & 4.08 & 3.1 & 3.05 & 3.05 \\
\hline & $\mathrm{C} 2$ & 2.7 & 4.09 & 3.7 & 3.49 & 3.49 \\
\hline & C3 & 2.96 & 4.64 & 3.6 & 3.51 & 3.5 \\
\hline & $\mathrm{C} 4$ & 4.01 & 4.69 & 4.3 & 4.07 & 4.06 \\
\hline Muste & CW01 & 0.739 & 7 & 2.92 & 2.79 & 2.78 \\
\hline and Patel & CW02 & 0.768 & 7.1 & 2.92 & 2.83 & 2.82 \\
\hline$(1997)[46]$ & CW03 & 0.813 & 7.16 & 2.98 & 2.9 & 2.89 \\
\hline MAPE & & & & & 3.23 & 3.48 \\
\hline RMSE & & & & & 0.13 & 0.14 \\
\hline MSD & & & & & -0.11 & -0.11 \\
\hline
\end{tabular}


Table 3. Comparison of the present model for bed shear velocities $(\mathrm{cm} / \mathrm{s})$ with experimental data from Coleman [43] for flow with suspended sediment.

\begin{tabular}{|c|c|c|c|c|c|c|c|c|}
\hline Run & $\begin{array}{c}M_{s} \\
(\mathrm{~kg})\end{array}$ & $\varphi \times 10^{-4}$ & $\begin{array}{c}h \\
(\mathrm{~cm})\end{array}$ & $b / h$ & $\begin{array}{l}h-\varepsilon \\
(\mathrm{cm})\end{array}$ & $\begin{array}{c}\boldsymbol{u}_{* b, m} \\
(\mathbf{c m} / \mathrm{s})\end{array}$ & $\begin{array}{c}u_{* b, 21} \\
(\mathrm{~cm} / \mathrm{s})\end{array}$ & $\begin{array}{c}u_{* b, G \& J} \\
(\mathbf{c m} / \mathbf{s})\end{array}$ \\
\hline 1 & 0 & 0 & 17.2 & 2.070 & 13.2 & 4.1 & 4.14 & 4.14 \\
\hline 2 & 0.91 & 2.12 & 17.1 & 2.082 & 12.0 & 4.1 & 4.09 & 4.14 \\
\hline 3 & 1.82 & 3.87 & 17.2 & 2.070 & 11.9 & 4.1 & 4.09 & 4.14 \\
\hline 4 & 2.73 & 5.87 & 17.1 & 2.082 & 12.8 & 4.1 & 4.12 & 4.14 \\
\hline 5 & 3.64 & 8.03 & 17.1 & 2.082 & 12.2 & 4.1 & 4.10 & 4.14 \\
\hline 6 & 4.54 & 9.76 & 17.0 & 2.094 & 11.9 & 4.1 & 4.08 & 4.13 \\
\hline 7 & 5.45 & 12.22 & 17.1 & 2.082 & 12.0 & 4.1 & 4.09 & 4.14 \\
\hline 8 & 6.36 & 14.37 & 17.3 & 2.058 & 13.0 & 4.1 & 4.14 & 4.15 \\
\hline 9 & 7.27 & 16.29 & 17.2 & 2.070 & 13.6 & 4.1 & 4.16 & 4.14 \\
\hline 10 & 8.18 & 18.56 & 17.1 & 2.082 & 13.4 & 4.1 & 4.15 & 4.14 \\
\hline 11 & 9.09 & 20.34 & 16.9 & 2.107 & 12.2 & 4.1 & 4.10 & 4.13 \\
\hline 12 & 10 & 21.69 & 17.3 & 2.058 & 13.4 & 4.1 & 4.15 & 4.15 \\
\hline 13 & 10.91 & 23.92 & 17.1 & 2.082 & 12.2 & 4.1 & 4.10 & 4.14 \\
\hline 14 & 11.82 & 25.64 & 17.1 & 2.082 & 12.2 & 4.1 & 4.10 & 4.14 \\
\hline 15 & 12.73 & 21.51 & 17.1 & 2.082 & 11.9 & 4.1 & 4.09 & 4.14 \\
\hline 16 & 13.64 & 28.39 & 17.1 & 2.082 & 11.2 & 4.1 & 4.06 & 4.14 \\
\hline 17 & 14.54 & 23.15 & 17.1 & 2.082 & 13.1 & 4.1 & 4.14 & 4.14 \\
\hline 18 & 15.45 & 28.56 & 17.2 & 2.070 & 13.1 & 4.1 & 4.14 & 4.14 \\
\hline 19 & 16.36 & 30.16 & 17.0 & 2.094 & 13.1 & 4.1 & 4.13 & 4.13 \\
\hline 20 & 17.27 & 31.24 & 17.0 & 2.094 & 12.6 & 4.1 & 4.11 & 4.13 \\
\hline 21 & 0 & 0 & 16.9 & 2.107 & 12.8 & 4.1 & 4.12 & 4.13 \\
\hline 22 & 0.91 & 1.80 & 17.0 & 2.094 & 12.2 & 4.1 & 4.10 & 4.13 \\
\hline 23 & 1.82 & 3.45 & 17.0 & 2.094 & 11.9 & 4.1 & 4.08 & 4.13 \\
\hline 24 & 2.73 & 5.27 & 16.9 & 2.107 & 12.2 & 4.1 & 4.10 & 4.13 \\
\hline 25 & 3.64 & 7.08 & 16.7 & 2.132 & 10.4 & 4.0 & 4.01 & 4.12 \\
\hline 26 & 4.54 & 8.84 & 17.1 & 2.082 & 13.0 & 4.1 & 4.13 & 4.14 \\
\hline 27 & 5.45 & 10.37 & 16.8 & 2.119 & 12.2 & 4.1 & 4.09 & 4.12 \\
\hline 28 & 6.36 & 12.00 & 17.0 & 2.094 & 12.2 & 4.1 & 4.10 & 4.13 \\
\hline 29 & 7.27 & 13.65 & 16.8 & 2.119 & 13.0 & 4.0 & 4.13 & 4.12 \\
\hline 30 & 8.18 & 15.00 & 16.8 & 2.119 & 13.7 & 4.1 & 4.16 & 4.12 \\
\hline 31 & 9.09 & 15.93 & 17.2 & 2.070 & 11.9 & 4.1 & 4.09 & 4.14 \\
\hline 32 & 0 & 0 & 17.3 & 2.058 & 13.1 & 4.1 & 4.14 & 4.15 \\
\hline 33 & 0.91 & 0.42 & 17.4 & 2.046 & 12.8 & 4.1 & 4.13 & 4.15 \\
\hline 34 & 1.82 & 0.71 & 17.2 & 2.070 & 13.1 & 4.1 & 4.14 & 4.14 \\
\hline 35 & 2.73 & 1.16 & 17.2 & 2.070 & 12.2 & 4.1 & 4.10 & 4.14 \\
\hline 36 & 3.64 & 1.89 & 17.1 & 2.082 & 12.2 & 4.1 & 4.10 & 4.14 \\
\hline 37 & 4.54 & 2.30 & 16.7 & 2.132 & 11.9 & 4.1 & 4.08 & 4.12 \\
\hline MAPE & & & & & & & 0.596 & 1.013 \\
\hline RMSE & & & & & & & 0.035 & 0.046 \\
\hline MSD & & & & & & & 0.016 & 0.041 \\
\hline
\end{tabular}


al. [48] and Knight and Sterling [49] provided interesting information about the secondary current structures and velocity distribution in trapezoidal channels and circular partially full channels, respectively. Khozani et al. [5] also studied the shear distribution in circular and trapezoidal channels. Tang and Knight [50] and Yang et al. [51] studied the flow pattern in compound open channels with regular and vegetated flows, respectively. These works can be handy for researchers who might be interested in applying our method to other crosssectional shapes or other flow regimes.

\section{Conclusion}

In the present paper, the channel cross-section was divided into parts using channel bisectors, at which shear force associated with secondary flows was zero and isovel orthogonal trajectories, at which shear force associated with velocity gradients was zero. The weight component of water in each of these subsections should be balanced either with bed or wall shear force. Based on these subsections, the minimum and maximum possible shear forces on walls and bed were calculated. The experimental data presented by various researchers lay well between the suggested maximum and minimum values. Considering the secondary flow structure, the share of the wall and bed from different subsections was calculated. An equation was finally derived for $\% S F_{w}$ and $\% S F_{b}$. Calculated wall and bed shear forces were in good agreement with experimental data with a mean absolute percentage error of less than $5 \%$.

The presented method can be used where the location of maximum velocity, $\varepsilon$, is known. $\varepsilon$ is available in experiments, or can be calculated directly from empirical dip equations or indirectly from velocity profile laws in case of no velocity data. The dependence of our equations on $\varepsilon$ makes it applicable to a wide range of steady-uniform open channel flows. In addition, the proposed equations were applied to flows with suspended sediment, at which the location of the maximum velocity was known with good agreement. While our equations work only for rectangular channels, the general concept is applicable to channels with different cross-sections.

\section{Nomenclature}

$\begin{array}{ll}A & \text { Area of the control surface } \\ A_{b \text { min }}, & \\ A_{w \text { min }}, & \text { Subsections on the channel cross- } \\ A_{1}, A_{2} & \text { section } \\ Q & \text { Discharge } \\ R^{2} & \text { Determination coefficient } \\ S & \text { Channel slope }\end{array}$

$\% S F_{b} \quad$ Percentage of the total shear force acting on the bed

$\% S F_{w} \quad$ Percentage of the total shear force acting on the walls

$V$ Velocity component of flow on $y-z$ plane

$b \quad$ Channel width

$\varepsilon \quad$ Depth of the maximum velocity

$h \quad$ Water depth

g Gravitational acceleration

$l \quad$ Length along the boundary of surface

n

$\eta_{1}, \eta_{2} \quad$ Parameters related to $A_{1}$ and $A_{2}$, respectively

$\rho \quad$ Density

$\varphi \quad$ Volumetric suspended sediment concentration

$\overline{\tau_{b}}, \overline{\tau_{w}} \quad$ Mean bed and wall shear stress, respectively

$u, \nu, w \quad$ Components of mean velocity in $x, y$, and $z$ directions, respectively

$u_{* b, G \& J} \quad$ Shear velocity predicted by Gou and Julien (2005)

$u_{* b, m} \quad$ Experimentally measured shear velocity

$\nu \quad$ Kinematic viscosity

$\nu_{t} \quad$ Turbulence viscosity

$u_{* b} \quad$ Mean shear velocity on bed

$\tau_{n x} \quad$ Shear stress in the flow direction $x$ applying on the plane perpendicular to $n$

$x \quad$ Longitudinal coordinate

$y \quad$ Vertical coordinate

z Spanwise coordinate

\section{References}

1. Hamedi, A. and Ketabdar, M. "Energy loss estimation and flow simulation in the skimming flow regime of stepped spillways with inclined steps and end sill: A numerical model", Int. J. Sci. Eng. Appl., 5(7), pp. 399-407 (2016).

2. Bardestani, S., Givehchi, M., Younesi, E., Sajjadi, S., Shamshirband, S., and Petkovic, D. "Predicting turbulent flow friction coefficient using ANFIS technique", Signal, Image Video Process, 11(2), pp. 341347 (2017).

3. Khozani, Z.S., Bonakdari, H., and Zaji, A.H. "Application of a soft computing technique in predicting the percentage of shear force carried by walls in a rectangular channel with non-homogeneous roughness", Water Sci. Technol., 73(1), pp. 124-129 (2016). 
4. Khozani, Z.S., Bonakdari, H., and Zaji, A.H. "Application of a genetic algorithm in predicting the percentage of shear force carried by walls in smooth rectangular channels", Measurement, 87, pp. 87-98 (2016).

5. Sheikh, Z. and Bonakdari, H. "Prediction of boundary shear stress in circular and trapezoidal channels with entropy concept", Urban Water J., 13(6), pp. 629-636 (2016).

6. Guo, J. and Julien, P.Y. "Shear stress in smooth rectangular open-channel flows", J. Hydraul. Eng., 131(1), pp. 30-37 (2005).

7. Termini, D. "Momentum transport and bed shear stress distribution in a meandering bend: Experimental analysis in a laboratory flume", Adv. Water Resour., 81, pp. 128-141 (2015).

8. Yang, S.-Q. and Lim, S.-Y. "Mechanism of energy transportation and turbulent flow in a 3D channel", J. Hydraul. Eng., 123(8), pp. 684-692 (1997).

9. Heydari, H., Zarrati, A.R., and Tabarestani, M.K. "Bed form characteristics in a live bed alluvial channel", Sci. Iran. Trans. A, Civ. Eng., 21(6), pp. 1773 (2014).

10. Cheng, N.-S. and Chua, L.H. "Comparisons of sidewall correction of bed shear stress in open-channel flows", J. Hydraul. Eng., 131(7), pp. 605-609 (2005).

11. Guo, J. and Julien, P.Y. "Boundary shear stress in smooth rectangular open-channels", In Advances in Hydraulics and Water Engineering, Proc. 13th IAHRAPD Congress, pp. 76-86 (2002).

12. Leighly, J.B., Toward a Theory of the Morphologic Significance of Turbulence in the Flow of Water in Streams, University of California Press (1932).

13. Chiu, C.-L. and Chiou, J.-D. "Structure of 3-D flow in rectangular open channels", J. Hydraul. Eng., 112(11), pp. 1050-1067 (1986).

14. Yang, S.-Q. "Interactions of boundary shear stress, secondary currents and velocity", Fluid Dyn. Res., 36(3), pp. 121-136 (2005).

15. Einstein, H.A. "Formulas for the transportation of bed load", Trans. ASCE Pap., 2140, pp. 561-597 (1942).

16. Keulegan, G.H., Laws of Turbulent Flow in Open Channels, National Bureau of Standards US (1938).

17. Schlichting, H. "Experimental investigation of the problem of surface roughness", Ingenieur-Archiv, 7(1), pp. 1-34 (1937).

18. Nezu, I. and Nakagawa, H. "Turbulence in openchannel flows", Vol. Monograph Series, Balkema, Rotterdam: International Association for Hydraulic Research (1993).

19. Henderson, F.M., Open Channel Flow, Macmillan (1996).

20. Maghrebi, M.F. and Rahimpour, M. "A simple model for estimation of dimensionless isovel contours in open channels", Flow Meas. Instrum., 16(6), pp. 347-352 (2005).
21. Houjou, K. and Ishii, C. "Calculation of boundary shear stress INi open channel flow", J. Hydrosclence Hydraul. Eng., 8(2), pp. 21-23 (1990).

22. De Cacqueray, N., Hargreaves, D.M., and Morvan, H.P. "A computational study of shear stress in smooth rectangular channels", J. Hydraul. Res., 47(1), pp. 5057 (2009).

23. Bonakdari, H., Larrarte, F., Lassabatere, L., and Joannis, C. "Turbulent velocity profile in fully-developed open channel flows", Environ. Fluid Mech., 8(1), pp. 1-17 (2008).

24. Nezu, I. and Rodi, W. "Open-channel flow measurements with a laser Doppler anemometer", J. Hydraul. Eng., 112(5), pp. 335-355 (1986).

25. Wang, X., Wang, Z.-Y., Yu, M., and Li, D. "Velocity profile of sediment suspensions and comparison of loglaw and wake-law", J. Hydraul. Res., 39(2), pp. 211217 (2001).

26. Chiu, C.-L. and Hsu, S.-M. "Probabilistic approach to modeling of velocity distributions in fluid flows", $J$. Hydrol., 316(1), pp. 28-42 (2006).

27. Yang, S.-Q., Tan, S.-K., and Lim, S.-Y. "Velocity distribution and dip-phenomenon in smooth uniform open channel flows", J. Hydraul. Eng., 130(12), pp. 1179-1186 (2004).

28. Knight, D.W., Demetriou, J.D., and Hamed, M.E. "Boundary shear in smooth rectangular channels", $J$. Hydraul. Eng., 110(4), pp. 405-422 (1984).

29. Gessner, F.B. and Jones, J.B. "On some aspects of fully-developed turbulent flow in rectangular channels", J. Fluid Mech., 23(04), pp. 689-713 (1965).

30. Tominaga, A., Nezu, I., Ezaki, K., and Nakagawa, H. "Three-dimensional turbulent structure in straight open channel flows", J. Hydraul. Res., 27(1), pp. 149173 (1989).

31. Zheng, Y. and Jin, Y.-C. "Boundary shear in rectangular ducts and channels", J. Hydraul. Eng., 124(1), pp. 86-89 (1998).

32. Jin, Y.-C., Zarrati, A.R. and Zheng, Y. "Boundary shear distribution in straight ducts and open channels", J. Hydraul. Eng., 130(9), pp. 924-928 (2004).

33. Cruff, R.W. "Cross-channel transfer of linear momentum in smooth rectangular channels", No. 1592-B. USGPO (1965).

34. Ghosh, S.N. and Roy, N. "Boundary shear distribution in open channel flow", J. Hydraul. Div., 96(HY4), Proc. Paper 7241, pp. 967-994 (1970).

35. Kartha, V.C. and Leutheusser, H.J. "Distribution of tractive force in open channels", J. Hydraul. Div., 96(HY 7), Proc. Paper 7415, pp. 1469-1483 (1970).

36. Knight, D.W. and Macdonald, J.A. "Hydraulic resistance of artificial strip roughness", J. Hydraul. Div., 105(6), pp. 675-690 (1979).

37. Knight, D.W. and Macdonald, J.A. "Open channel flow with varying bed roughness", J. Hydraul. Div., 105(9), pp. 1167-1183 (1979). 
38. Knight, D.W. "Boundary shear in smooth and rough channels", J. Hydraul. Div., 107(7), pp. 839-851 (1981).

39. Myers, W.R.C. "Momentum transfer in a compound channel", J. Hydraul. Res., 16(2), pp. 139-150 (1978).

40. Seckin, G., Seckin, N., and Yurtal, R. "Boundary shear stress analysis in smooth rectangular channels", Can. J. Civ. Eng., 33(3), pp. 336-342 (2006).

41. Xie, Q. "Turbulent flows in non-uniform open channels: experimental measurements and numerical modelling", PhD Thesis, Department of Civil Engineering, The University of Queensland (1998).

42. Chanson, H. "Boundary shear stress measurements in undular flows: Application to standing wave bed forms", Water Resour. Res., 36(10), pp. 3063-3076 (2000).

43. Coleman, N.L. "Effects of suspended sediment on the open-channel velocity distribution", Water Resour. Res., 22(10), pp. 1377-1384 (1986).

44. Lyn, D.A. "Turbulence and turbulent transport in sediment-laden open-channel flows", Dissertation (Ph.D.), California Institute of Technology (1987).

45. Lyn, D.A. "Regression residuals and mean profiles in uniform open-channel flows", J. Hydraul. Eng., 126(1), pp. 24-32 (2000).

46. Muste, M. and Patel, V.C. "Velocity profiles for particles and liquid in open-channel flow with suspended sediment", J. Hydraul. Eng., 123(9), pp. 742-751 (1997).

47. Coleman, N.L. "Velocity profiles with suspended sediment", J. Hydraul. Res., 19(3), pp. 211-229 (1981).

48. Knight, D.W., Omran, M., and Tang, X. "Modeling depth-averaged velocity and boundary shear in trapezoidal channels with secondary flows", J. Hydraul. Eng., 133(1), pp. 39-47 (2007).

49. Knight, D.W. and Sterling, M. "Boundary shear in circular pipes running partially full", J. Hydraul. Eng., 126(4), pp. 263-275 (2000).
50. Tang, X. and Knight, D.W. "Lateral depth-averaged velocity distributions and bed shear in rectangular compound channels", J. Hydraul. Eng., 134(9), pp. 1337-1342 (2008).

51. Yang, K., Cao, S., and Knight, D.W. "Flow patterns in compound channels with vegetated floodplains", $J$. Hydraul. Eng., 133(2), pp. 148-159 (2007).

\section{Biographies}

Sasan Tavakkol is a PhD candidate in the University of Southern California (USC), Los Angeles, USA. His $\mathrm{PhD}$ research concerns the development of an interactive real-time software for simulation and visualization of coastal waves. This software, called Celeris, is then employed along with crowdsourced data in disaster to increase situational awareness. He received his second MSc in computer science from USC where his research project was concerned with prioritizing crowdsourced data. Sasan obtained his BSc and first MSc from Amirkabir University of Technology (AUT) with the highest GPA. His research topics at AUT include Smoothed Particle Hydrodynamics (SPH) and open channel flows.

Amir Reza Zarrati is a Professor of Hydraulic Engineering at the Department of Civil and Environmental Engineering, Amirkabir University of Technology Tehran, Iran. He has more than 20 years of teaching and research experience. His main area of research is numerical and physical modelling of flow behaviour in hydraulic structures and rivers. He is also interested in scouring phenomenon and methods of its control. Dr. Zarrati has also a long history of collaboration with the industry and has been a senior consultant in a number of large dams in the country. $\mathrm{He}$ is a member of Iranian Hydraulic Association (IHA) and International Association of Hydraulic Research (IAHR). 